\title{
STARD3/MLN64 is Striving at Membrane Contact Sites: Intracellular Cholesterol Trafficking for Steroidogenesis in Human Placental Cells
}

\author{
Atsuki Nara, Tohru Komiya \\ Nagahama Institute of Bioscience and Technology, Shiga, Japan \\ Email address: \\ a_nara@nagahama-i-bio.ac.jp (A. Nara),t_komiya@nagahama-i-bio.ac.jp (T. komiya)
}

To cite this article:

Atsuki Nara, Tohru Komiya. STARD3/MLN64 is Striving at Membrane Contact Sites: Intracellular Cholesterol Trafficking for Steroidogenesis in Human Placental Cells. American Journal of Life Sciences. Special Issue: Biology and Medicine of Peptide and Steroid Hormones. Vol. 3, No. 3-2, 2015, pp. 48-52. doi: 10.11648/j.ajls.s.2015030302.19

\begin{abstract}
Steroid hormone synthesis begins with the conversion of cholesterol into pregnenolone by the enzyme cytochrome P450scc in mitochondria. The cholesterol used to synthesize pregnenolone is derived mainly from endocytosed LDL cholesterol. How this LDL cholesterol is transported to mitochondria in the human placenta is not well understood. Recent work has focused on how STARD3/MLN64 controls cholesterol trafficking. The STARD3 protein has a START domain that associates with cholesterol. Why STARD3 is distributed mainly to late endosomes but not to mitochondria is an obstacle to understanding the early steps in steroidogenesis. STARD3 can bind the ER protein VAP and contribute to ER-late endosome MCS formation. In this review, recent progress on STARD3 functions suggests possible models to explain how cholesterol could transit to mitochondria via MCS.
\end{abstract}

Keywords: STARD3, steroidogenesis, endosome, mitochondria, cholesterol, membrane contact site

\section{Abbreviations}

ABCA, ATP-binding cassette class A; ER, endoplasmic reticulum; FFAT, two phenylalanines in an acidic tract; LDL, low-density lipoprotein; LBPA, lysobisphosphatidic acid; MAM, mitochondria-associated ER membrane; MENTHO, MLN64 N-terminal domain homologue; MCS, membrane contact sites; MLN64, metastatic lymph node, code 64; NPC, Niemann-Pick type C; ORP, oxysterol-binding protein-related protein; StAR, steroidogenic acute regulatory protein; START, StAR-related lipid transfer; VAP, VAMP-associated protein.

\section{Introduction}

The cholesterol required for steroidogenesis in human cells is mostly derived from low-density lipoprotein (LDL) absorbed by receptor-mediated endocytosis. LDL, which binds to its receptor on the plasma membrane, is endocytosed and delivered to early endosomes. The LDL-receptor complex dissociates within these compartments; while the receptor is returned to the plasma membrane for reuse, the LDL is transported to late endosomes/lysosomes for degradation. Cholesterol hydrolyzed by acid lipase in lysosomes is subsequently delivered to several compartments [1]. Cholesterol content differs from one intracellular compartment to another. Therefore, cholesterol trafficking from endosomes to other intracellular compartments, such as mitochondria, should be strictly regulated.

Steroid hormone synthesis begins with the conversion of cholesterol into pregnenolone production by the enzyme cytochrome P450scc in mitochondria [2, 3]. As "steroid hormone factories," mitochondria receive cholesterol for making pregnenolone that is subsequently used to synthesize hormones such as aldosterone and cortisol. In almost all steroidogenic cells, cholesterol transfer to the mitochondria is mediated by steroidogenic acute regulatory protein (StAR), which functions on the surface of the outer mitochondrial membrane $[4,5]$. StAR is crucial for steroidogenesis in several types of mammalian cells and StAR over-expression increases steroid hormone synthesis in both steroidogenic and non-steroidogenic cells [6-8]. Despite this, steroidogenesis can also be carried out in a StAR-independent manner, such as in the human placenta where StAR is not expressed [9].

StAR-independent steroidogenesis in the placenta may substitute STARD3/MLN64 for StAR and several studies have revealed that STARD3 has a steroidogenic activity 
similar to that of StAR [10]. One notable difference between StAR and STARD3 is their intracellular distribution. In previous reports, STARD3 has been found to localize mainly to endosomes but not to mitochondria [11-14]. Since several lipid-binding proteins are found in endosomes such as NPC1, NPC2, ABCA3, and STARD3, it is important to determine which protein is crucial for trafficking cholesterol from endosomes to mitochondria. Recent work has shown that STARD3 functions in cholesterol trafficking to the mitochondria even in the absence of NPC1 function [15]. Thus, STARD3 could play a key regulatory role in cholesterol trafficking to mitochondria in the placenta although little is currently known about either how cholesterol is transferred to mitochondria or roles that STARD3 might play in mitochondrial cholesterol trafficking. This motivated us to investigate how STARD3 regulates cholesterol trafficking to mitochondria in human placental cells. In this review, we briefly summarize prior studies and consider possible mechanisms for STARD3 functions in cholesterol trafficking.

\section{The Function of the StAR-Related Lipid Transfer (START) Domain in STARD3}

The StAR-related lipid transfer (START) domain is made up of approximately 210 amino-acid residues and has been implicated in intracellular membrane transport, lipid metabolism, and cell signaling [16-19]. The late endosomal membrane-spanning STARD3 protein contains a START domain that faces the cytoplasm. Computational modeling and analysis of its three-dimensional structure revealed that the START domain can bind cholesterol in a similar manner to StAR [20]. Moreover, the C-terminal amino-acid sequence of STARD3, which contains the START domain, is about $54 \%$ similar to that of StAR [8]. Bacterially expressed recombinant STARD3 lacking its N-terminal 218 amino acids (N-218) appears to possess pregnenolone-generating activity as measured by an in vitro assay system using purified mitochondria [21]. Cells carrying a plasmid expressing N-218 STARD3 also produce pregnenolone at high levels $[12,22]$. START-STARD3-GFP, however, displays a diffuse distribution throughout the cytoplasm [12]. This observation casts doubt on whether the START domain fragment directly recruits and transports cholesterol to the mitochondria. Trypsin-catalyzed proteolytic analysis, in contrast, indicates differential cholesterol binding by each START domain [23]. Thus, an alternative idea is that the START domains of StAR and STARD3 differ slightly in their ability to transport cholesterol to mitochondria.

A cleaved START domain fragment of STARD3 has been shown to be present in the human placenta [10]. Recent reports suggest that STARD3 is modified by a mitochondrial protease in the human placenta [24]. Although a mitochondrial leader sequence of StAR is also processed, this processing is not essential for pregnenolone synthesis [5]. The processed STARD3, like N-218, can activate progesterone synthesis in a purified placental mitochondria fraction, which is reminiscent of Tuckey's study [21]. It is still unclear, however, how STARD3, an endosomal membrane protein, interacts with the mitochondrial protease.

\section{STARD3-Interacting Proteins}

Several recent works have uncovered physical interactions between STARD3 and other proteins. The first protein identified as a STARD3 interactor is MENTHO/STARD3L (MLN64 NH2-terminal domain homologue), also an endosomal membrane protein [25]. STARD3 and MENTHO form both homo- and hetero-oligomers [26], and these dimerizing interactions presumably provide discrete, specific membrane regions such as cholesterol-rich membrane rafts. Such regions may play a role in cholesterol egress from endosomes to other compartments.

STARD3 has been observed to co-localize specifically with a cholesterol transporter, ABCA3, in endosomes [14], which suggests that $\mathrm{ABCA} 3$ may mediate cholesterol release together with STARD3-MENTHO and/or MENTHO complexes.

The 14-3-3 protein complex, which is active in cellular signaling involving phosphorylation [27], can bind to STARD3 and regulate STARD3 movement from endosomes to the plasma membrane [28].

Interestingly, VAP proteins in the ER, but not in endosomes, interact with STARD3 via the FFAT motif and have been proposed to act as a molecular tether between late endosomes and the ER [13]. Nevertheless, the physiological significance of the tethering remains unclear.

\section{STARD3 Localization}

Previous work has shown that STARD3 is a late endosomal protein [11, 12, 29]. In MCF7 human breast cancer cells transfected with a STARD3 plasmid, STARD3 co-localizes with late endosome markers such as Cathepsin D, CD63, and LBPA [11]. Electron microscopic analysis revealed that STARD3 localizes to the limiting membrane of the late endosomes [11]. More than half of intracellular cholesterol is present in late endosomes [30] where many cholesterol-binding proteins, such as NPC1, NPC2, and ORP1L, are thought to control cholesterol transport to other compartments. Among these proteins, it is notable that functional NPC1 is not required for cholesterol release by STARD3 to the mitochondria [15]. Moreover, STARD3 localizes to ABCA3-positive late endosomes, but does not co-localize with NPC1 to ORP1L-positive late endosomes [14]. van der Kant et al. showed that endocytosed cargoes destined for lysosomes were delivered to NPC1-containing late endosomes via STARD3-containing compartments. These results suggest, intriguingly, that late endosomes are further subdivided functionally into STARD3-containing 'early' late endosomes and NPC1-containing compartment 'late' late endosomes. Furthermore, it is possible that the 'early' late endosomes transfer cholesterol to mitochondria. Another 
intriguing possibility is that STARD3, together with ABCA3, can be recycled between 'early' late endosomes and the plasma membrane. If so, STARD3 should be present not only in late endosomes but also in the plasma membrane.

\section{Two Models for Cholesterol Trafficking from Endosomes to Mitochondria}

One model for how STARD3 mediates cholesterol trafficking in the placenta from late endosomes to mitochondria is illustrated in Figure 1. In eukaryotic cells, lipid transfer between organelles occurs at membrane contact sites (MCS) where each limiting membrane is closely apposed [31-34]. MCS provide regions for some protein-protein interactions and for exchanging signals and small molecules, such as lipids and ions. Both cholesterol-binding proteins STARD3 and MENTHO can bind the ER protein VAP. Hence, it is possible that cholesterol from late endosomes is transported to the ER via endosome-ER MCS and subsequently to mitochondria via the mitochondria-associated ER membrane (MAM) [35]. STARD3 has been proposed to sort cholesterol to the ER. Interestingly, the cholesterol content in the ER is lower (approximately 1\%) than in either the plasma membrane or endosomes [36]. In addition, transfer from lysosomes to the plasma membrane takes less than $2 \mathrm{~min}$ [37]. Hence, cholesterol may pass through the ER rapidly and subsequently transit to mitochondria. Since the ER associates with both late endosomes and mitochondria, such indirect apposition may contribute to the cholesterol transfer.

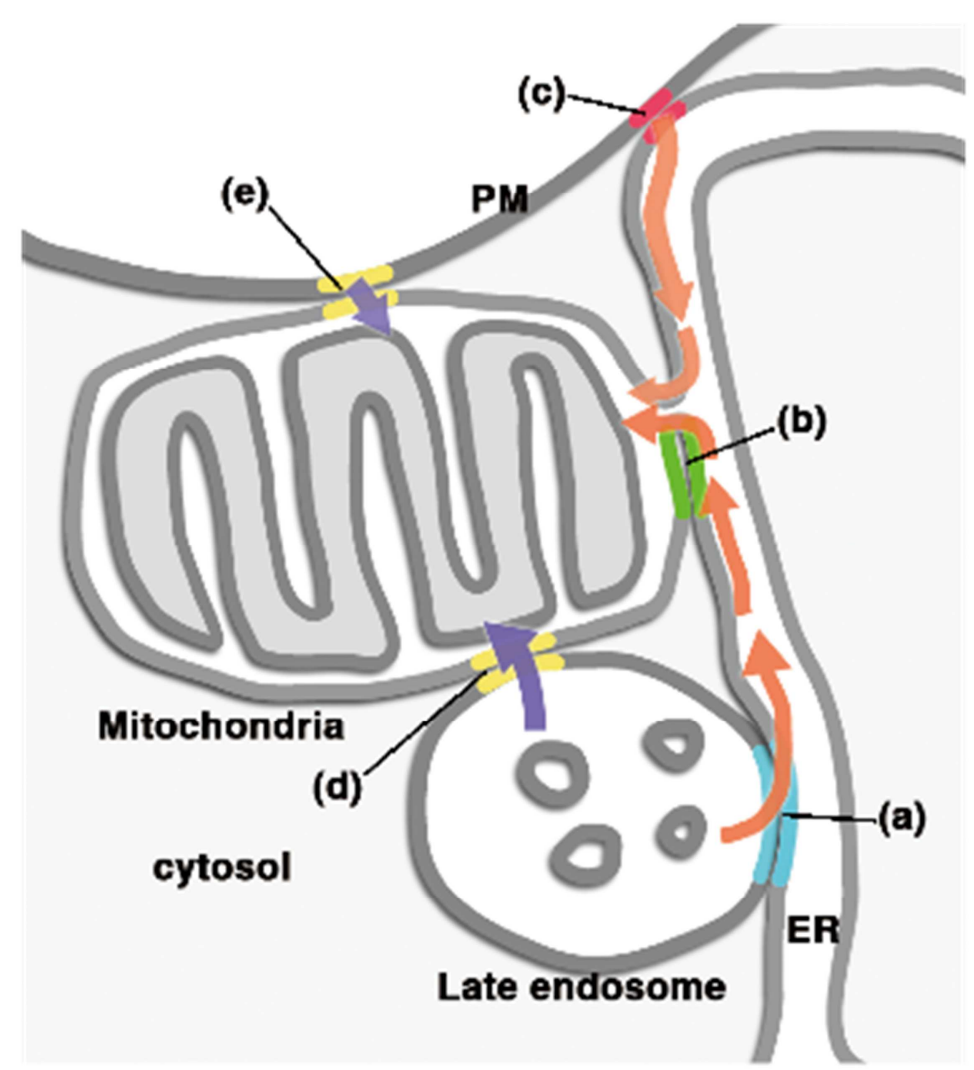

Figure 1. Possible model for cholesterol flow to mitochondria. (a) The late endosome protein STARD3 binds to the ER proteins VAP, forming ER-late endosome MCS (blue) where cholesterol could be transferred (red arrow). (b) Cholesterol is sorted to mitochondria via either ER-mitochondria MCS (green) or the MAM (red arrow). (c) STARD3 also localizes to the PM; therefore, cholesterol in PM could be sorted to the ER via the ER-PM MCS (red). Provided that the outer membranes of mitochondria and the limiting membranes of late endosomes (d), and PM (e) come into close contact as they do at MCS, cholesterol from these compartments would be transferred directly to the mitochondria (purple arrows).

Alternatively, 'late endosome-mitochondria' MCS may form that mediate cholesterol sorting. We detected this close membrane contact by electron microscopy (unpublished results). Early endosomes, which are the first to receive endocytosed LDL, are far from mitochondria and probably not where cholesterol sorting occurs (unpublished results). The STARD3-VAP complex, probably acting as a tether, could contribute to MCS formation. Since some lipid exchange at MCS does not appear to require START domain-containing proteins, other lipid transporter-like $\mathrm{ABCAs}$ such as $\mathrm{ABCA} 3$ may mediate cholesterol transport directly from late endosomes to mitochondria [34]. In the placenta, rapid progesterone production is needed in order to maintain pregnancy. We therefore suspect that 'cholesterol-loaded' late endosomes directly sort cholesterol to mitochondria. Since STARD3 has also been found in the plasma membrane, it could help transport cholesterol on the plasma membrane directly to mitochondria or via the ER (Figure 1). These 
hypotheses are not mutually exclusive and require further investigation.

\section{Concluding Remarks}

Although recent work has begun to elucidate the role of STARD3 in steroidogenesis, how cholesterol is transported from one compartment to another remains to be clarified. To elucidate any speculation concerning cholesterol trafficking, we have reviewed specific parts of selected, recent studies concerning STARD3. We apologize to any researchers whose work could not be cited here. We look forward to discussing their work in the future.

\section{Acknowledgements}

We are supported by Collaborative Research Grants for Nagahama Institute of Bio-science and Technology.

\section{References}

[1] E. Ikonen, "Cellular cholesterol trafficking and compartmentalization," Nat. Rev. Mol. Cell Biol., 2008, 9, pp. 125-138.

[2] W.L. Miller, "Steroid hormone synthesis in mitochondria," Mol. Cell. Endocrinol., 2013, 379, pp. 62-73.

[3] W.L. Miller, R.J. Auchus, "The molecular biology, biochemistry, and physiology of human steroidogenesis and its disorders," Endocr. Rev., 2011, 32, pp. 81-151.

[4] J.F. Strauss, 3rd, C.B. Kallen, L.K. Christenson et al., "The steroidogenic acute regulatory protein (StAR): a window into the complexities of intracellular cholesterol trafficking," Recent Prog. Horm. Res., 1999, 54, pp. 369-394; discussion 394-365.

[5] H.S. Bose, V.R. Lingappa, W.L. Miller, "Rapid regulation of steroidogenesis by mitochondrial protein import," Nature, 2002, 417, pp. 87-91.

[6] B.J. Clark, J. Wells, S.R. King, D.M. Stocco, "The purification, cloning, and expression of a novel luteinizing hormone-induced mitochondrial protein in MA-10 mouse Leydig tumor cells. Characterization of the steroidogenic acute regulatory protein (StAR)," J. Biol. Chem., 1994, 269, pp. 28314-28322.

[7] D. Lin, T. Sugawara, J.F. Strauss, 3rd et al., "Role of steroidogenic acute regulatory protein in adrenal and gonadal steroidogenesis," Science, 1995, 267, pp. 1828-1831.

[8] W.L. Miller, H.S. Bose, "Early steps in steroidogenesis: intracellular cholesterol trafficking," J. Lipid Res., 2011, 52, pp. 2111-2135.

[9] T. Sugawara, J.A. Holt, D. Driscoll et al., "Human steroidogenic acute regulatory protein: functional activity in COS-1 cells, tissue-specific expression, and mapping of the structural gene to $8 \mathrm{p} 11.2$ and a pseudogene to chromosome 13," Proc. Natl. Acad. Sci. U. S. A., 1995, 92, pp. 4778-4782.

[10] H.S. Bose, R.M. Whittal, M.C. Huang, M.A. Baldwin, W.L. Miller, "N-218 MLN64, a protein with StAR-like steroidogenic activity, is folded and cleaved similarly to StAR," Biochemistry, 2000, 39, pp. 11722-11731.

[11] F. Alpy, M.E. Stoeckel, A. Dierich et al., "The steroidogenic acute regulatory protein homolog MLN64, a late endosomal cholesterol-binding protein," J. Biol. Chem., 2001, 276, pp. 4261-4269.

[12] M. Zhang, P. Liu, N.K. Dwyer et al., "MLN64 mediates mobilization of lysosomal cholesterol to steroidogenic mitochondria," J. Biol. Chem., 2002, 277, pp. 33300-33310.

[13] F. Alpy, A. Rousseau, Y. Schwab et al., "STARD3 or STARD3NL and VAP form a novel molecular tether between late endosomes and the ER," J. Cell Sci., 2013, 126, pp. 5500-5512.

[14] R. van der Kant, I. Zondervan, L. Janssen, J. Neefjes, "Cholesterol-binding molecules MLN64 and ORP1L mark distinct late endosomes with transporters ABCA3 and NPC1," J. Lipid Res., 2013, 54, pp. 2153-2165.

[15] M. Charman, B.E. Kennedy, N. Osborne, B. Karten, "MLN64 mediates egress of cholesterol from endosomes to mitochondria in the absence of functional Niemann-Pick Type C1 protein," J. Lipid Res., 2010, 51, pp. 1023-1034.

[16] R.E. Soccio, J.L. Breslow, "StAR-related lipid transfer (START) proteins: mediators of intracellular lipid metabolism," J. Biol. Chem., 2003, 278, pp. 22183-22186.

[17] F. Alpy, C. Tomasetto, "Give lipids a START: the StAR-related lipid transfer (START) domain in mammals," J. Cell Sci., 2005, 118, pp. 2791-2801.

[18] G. D'Angelo, M. Vicinanza, M.A. De Matteis, "Lipid-transfer proteins in biosynthetic pathways," Curr. Opin. Cell Biol., 2008, 20, pp. 360-370.

[19] B. Mesmin, B. Antonny, G. Drin, "Insights into the mechanisms of sterol transport between organelles," Cell. Mol. Life Sci., 2013, 70, pp. 3405-3421.

[20] M. Murcia, J.D. Faraldo-Gomez, F.R. Maxfield, B. Roux, "Modeling the structure of the StART domains of MLN64 and StAR proteins in complex with cholesterol," J. Lipid Res., 2006, 47, pp. $2614-2630$.

[21] R.C. Tuckey, H.S. Bose, I. Czerwionka, W.L. Miller, "Molten globule structure and steroidogenic activity of N-218 MLN64 in human placental mitochondria," Endocrinology, 2004, 145, pp. 1700-1707.

[22] H. Watari, F. Arakane, C. Moog-Lutz et al., "MLN64 contains a domain with homology to the steroidogenic acute regulatory protein (StAR) that stimulates steroidogenesis," Proc. Natl. Acad. Sci. U. S. A., 1997, 94, pp. 8462-8467.

[23] J. Reitz, K. Gehrig-Burger, J.F. Strauss, 3rd, G. Gimpl, "Cholesterol interaction with the related steroidogenic acute regulatory lipid-transfer (START) domains of StAR (STARD1) and MLN64 (STARD3)," Febs j, 2008, 275, pp. 1790-1802.

[24] M. Esparza-Perusquia, S. Olvera-Sanchez, O. Flores-Herrera et al., "Mitochondrial proteases act on STARD3 to activate progesterone synthesis in human syncytiotrophoblast," Biochim. Biophys. Acta, 2015, 1850, pp. 107-117.

[25] F. Alpy, C. Tomasetto, "MLN64 and MENTHO, two mediators of endosomal cholesterol transport," Biochem. Soc. Trans., 2006, 34, pp. 343-345. 
[26] F. Alpy, V.K. Latchumanan, V. Kedinger et al., "Functional characterization of the MENTAL domain," J. Biol. Chem., 2005, 280, pp. 17945-17952.

[27] G. Tzivion, J. Avruch, "14-3-3 proteins: active cofactors in cellular regulation by serine/threonine phosphorylation," J. Biol. Chem., 2002, 277, pp. 3061-3064.

[28] A. Liapis, F.W. Chen, J.P. Davies, R. Wang, Y.A. Ioannou, "MLN64 transport to the late endosome is regulated by binding to 14-3-3 via a non-canonical binding site," PLoS One, 2012, 7 , pp. e34424.

[29] M. Holtta-Vuori, F. Alpy, K. Tanhuanpaa et al., "MLN64 is involved in actin-mediated dynamics of late endocytic organelles," Mol. Biol. Cell, 2005, 16, pp. 3873-3886.

[30] W. Mobius, E. van Donselaar, Y. Ohno-Iwashita et al., "Recycling compartments and the internal vesicles of multivesicular bodies harbor most of the cholesterol found in the endocytic pathway," Traffic, 2003, 4, pp. 222-231.

[31] T. Levine, C. Loewen, "Inter-organelle membrane contact sites: through a glass, darkly," Curr. Opin. Cell Biol., 2006, 18, pp. 371-378.

[32] M. Lebiedzinska, G. Szabadkai, A.W. Jones, J. Duszynski, M.R. Wieckowski, "Interactions between the endoplasmic reticulum, mitochondria, plasma membrane and other subcellular organelles," Int. J. Biochem. Cell Biol., 2009, 41, pp. 1805-1816.

[33] Y. Elbaz, M. Schuldiner, "Staying in touch: the molecular era of organelle contact sites," Trends Biochem. Sci., 2011, 36, pp. 616-623.

[34] A. Toulmay, W.A. Prinz, "Lipid transfer and signaling at organelle contact sites: the tip of the iceberg," Curr. Opin. Cell Biol., 2011, 23, pp. 458-463.

[35] T. Hayashi, R. Rizzuto, G. Hajnoczky, T.P. Su, "MAM: more than just a housekeeper," Trends Cell Biol., 2009, 19, pp. 81-88.

[36] Y. Lange, J. Ye, M. Rigney, T.L. Steck, "Regulation of endoplasmic reticulum cholesterol by plasma membrane cholesterol," J. Lipid Res., 1999, 40, pp. 2264-2270.

[37] D.L. Brasaemle, A.D. Attie, "Rapid intracellular transport of LDL-derived cholesterol to the plasma membrane in cultured fibroblasts," J. Lipid Res., 1990, 31, pp. 103-112. 